\title{
Performance of the DGM for the Linearized Euler Equations With Non-Uniform Mean-Flow
}

\author{
Michael Williamschen*, Gwénaël Gabard ${ }^{\dagger}$, and Hadrien Bériot ${ }^{\ddagger}$
}

\begin{abstract}
A dispersion analysis of the fully-discrete, nodal discontinuous Galerkin method (DGM) for the solution of the time-domain linearized Euler equations (LEE) is performed. Two dispersion analysis methods are developed, considering both uniform and non-uniform mean-flow effects. Convergence studies are performed for the dispersion, dissipation, and nodal solution errors of the acoustic, entropy, and vorticity modes. The accuracy and stability of the DGM are analyzed in the context of aeroacoustic applications, and guidelines are proposed for the choice of optimal discretizations. Computational costs are estimated for a model problem and related to the choice of the element size, polynomial order, and time step. Results indicate that temporal error can become a dominant source of error for high accuracy requirements and long distance wave propagation. The stability of the scheme is analyzed for a shear layer mean flow profile. Aliasing-type errors are found to contribute to the formation of numerical instabilities which are further strengthened by increases in the polynomial order.
\end{abstract}

\section{Introduction}

Aeroacoustics problems, such as turbofan noise predictions, require both high accuracy, for far-field sound radiation, and complex geometry representation, for the support of complex geometries commonly encountered in modern engine designs. Nodal Discontinuous Galerkin Methods (DGM) have proven to be popular schemes for the solution of hyperbolic problems where both high-order accuracy and representation of complex geometries are required. The use of the quadrature-free formulation, developed by Atkins and Shu, ${ }^{1}$ makes the DGM competitive, in terms of computational efficiency, with other popular schemes. However, reducing the computational cost is still a priority, especially for the solution of complex three-dimensional (3D) problems where far-field sound radiation is required. Techniques to reduce computational costs can include multi-rate time stepping, mesh and/or order refinement ( $h$ and $p$-refinement), or new time integration strategies. Common to all techniques is that they require a priori knowledge of the accuracy and stability of the scheme to determine the appropriate time step, element order, or mesh size.

High-order accuracy estimates of the DGM for wave propagation problems have been studied extensively by the use of dispersion analysis techniques. ${ }^{2,3,4,5,6,7}$ The spectral error for the semi-discrete scheme and $h \rightarrow 0$ is found to converge at $h^{2 p+3}$, for the absolute dispersion error, and $h^{2 p+1}$ for the dissipation error. The linear stability of the fully-discrete scheme including stable time step estimates and influence of the element shape have been evaluated for the scalar advection equation..$^{8,9}$

However, for aeroacoustics applications, problems are often governed by the linearized Euler equations (LEE). ${ }^{10,11,12,13,14,15,16}$ Therefore, while dispersion analyses gave estimates of the performance of the DGM for model problems, considering the full form of the linearized Euler Equations may provide additional insight into the performance of the scheme, specifically for aeroacoustics problems. These problems typically involve highly non-uniform mean flows and the effects of this on the stability and accuracy of the scheme have not yet been fully realized. Therefore, two dispersion analysis techniques are developed herein, one following traditional dispersion analysis techniques ${ }^{2,3}$ with the LEE, and a new technique allowing non-uniform mean flow.

The paper is outlined as follows: Section 1 displays the linearized Euler equations and the formulation of the quadrature-free DGM. Section 2 illustrates the semi and fully-discrete dispersion analysis for the LEE

*PhD Candidate, University of Southampton ISVR

${ }^{\dagger}$ Associate Professor, University of Southampton ISVR and Senior AIAA member

${ }^{\ddagger}$ Siemens PLM 
in the presence of a uniform mean flow, then attempts to show the accuracy of the scheme and develops guidelines for simulation parameters to minimize the computational cost. Section 3 develops the LEE dispersion analysis method for a non-uniform mean flow, then uses the analysis to study the formation of instabilities. Section 4 summarizes the findings of this paper and sets objectives for future work.

\section{Governing equations and the nodal discontinuous Galerkin method}

In aeroacoustics applications, the linearized Euler equations (LEE) govern the propagation of small acoustic and hydrodynamic disturbances. In this section, the LEE are defined and then discretized with the nodal discontinuous Galerkin method.

\section{A. The linearized Euler equations}

Two-dimensional sound propagation through a non-uniform fluid flow can be approximated by linearizing the conservative form of the Euler equations about a mean flow, resulting in the linearized Euler equations,

$$
\frac{\partial \mathbf{U}}{\partial t}+\frac{\partial \mathbf{F}}{\partial x}+\frac{\partial \mathbf{G}}{\partial y}=\mathbf{S}
$$

for the fluctuating quantities, $\mathbf{U}=\left[\rho^{\prime},(\rho u)^{\prime},(\rho v)^{\prime}, \pi^{\prime}\right]^{\mathrm{T}}$, where $\rho^{\prime},(\rho u)^{\prime},(\rho v)^{\prime}$, and $\pi^{\prime}$ are the perturbations of the fluid density, $x$-momentum, $y$-momentum, and pressure ratio, respectively. The pressure ratio, $\pi^{\prime}$, similar to the variable used by Goldstein, ${ }^{17}$ is defined as $\pi^{\prime}=\left(\frac{p^{\prime}}{p_{\infty}}\right)^{\frac{1}{\gamma}}$, where $p^{\prime}$ is the pressure perturbation, $p_{\infty}$ is a reference pressure for normalization, and $\gamma$ is the ratio of specific heats of the fluid. The fluxes in the $x$ and $y$-directions are given by

$$
\mathbf{F}=\left[\begin{array}{cccc}
0 & 1 & 0 & 0 \\
-u_{\circ}^{2} & 2 u_{\circ} & 0 & \rho_{\circ} c_{\circ}^{2} \pi_{\circ} \\
-u_{\circ} v_{\circ} & v_{\circ} & u_{\circ} & 0 \\
-u_{\circ} \pi_{\circ} / \rho_{\circ} & \pi_{\circ} / \rho_{\circ} & 0 & u_{\circ}
\end{array}\right] \mathbf{U}, \quad \mathbf{G}=\left[\begin{array}{cccc}
0 & 0 & 1 & 0 \\
-u_{\circ} v_{\circ} & v_{\circ} & u_{\circ} & 0 \\
-v_{\circ}^{2} & 0 & 2 v_{\circ} & \rho_{\circ} c_{\circ}^{2} / \pi_{\circ} \\
-v_{\circ} \pi_{\circ} / \rho_{\circ} & 0 & \rho_{\circ} / \pi_{\circ} & v_{\circ}
\end{array}\right] \mathbf{U},
$$

where $\rho_{\circ}, u_{\circ}, v_{\circ}, c_{\circ}$, and $\pi_{\circ}$ are the density, $x$-velocity, $y$-velocity, sound speed, and pressure ratio of the mean flow field around which the Euler equations are linearized. The source term, $\mathbf{S}$, contains any sources of mass, momentum, or pressure perturbations in the domain, e.g. sources of sound. The following portion of the paper will refer to the fluctuating quantities as $\rho, \rho u, \rho v$, and $\pi$, dropping the primes for clarity.

It is well known that solutions to Equation (1) are in the form of acoustic, entropy, and vorticity modes. ${ }^{18}$ The presence of a geometry can scatter entropy and vorticity fluctuations into acoustic waves. Strongly nonuniform mean flows, such as shear layers, can excite the vorticity mode leading to the growth of instabilities. ${ }^{19}$

\section{B. The nodal discontinuous Galerkin method}

This work employs the quadrature-free, Nodal discontinuous Galerkin method developed by Atkins and Shu ${ }^{1}$ to solve the conservation form of the LEE in Equation (1). The quadrature-free method can significantly reduce memory storage requirements for triangular elements with constant Jacobian. Application of the quadrature-free method to the 2D LEE follows below.

Defining a triangular tessellation, $\Omega \subset \mathbb{R}^{2}$, an element, $D_{k} \in \Omega$, and requiring that the residual of Equation (1) is orthogonal to all test functions $\phi \in \mathcal{L}_{p}$ in element $D_{k}$, where $\mathcal{L}_{p}=\left\{\phi_{i}, i=1,2, \ldots, N_{p}\right\}$ is the space of Lagrange interpolating polynomials of order $p$,

$$
\int_{D_{k}}\left(\frac{\partial \mathbf{U}_{k}}{\partial t}+\frac{\partial \mathbf{F}_{k}}{\partial x}+\frac{\partial \mathbf{G}_{k}}{\partial y}-\mathbf{S}_{k}\right) \phi_{i} \mathrm{~d} D_{k}=0, \quad 1 \leq i \leq N_{p} .
$$

Integrating by parts twice, the strong formulation of the DGM becomes

$$
\int_{D_{k}} \frac{\partial \mathbf{U}_{k}}{\partial t} \phi_{i} \mathrm{~d} D_{k}=\int_{D_{k}} \mathbf{S}_{k} \phi_{i}-\left(\frac{\partial \mathbf{F}_{k}}{\partial x}+\frac{\partial \mathbf{G}_{k}}{\partial y}\right) \phi_{i} \mathrm{~d} D_{k}+\sum_{b=1}^{3} \int_{\Gamma_{b, k}}\left(\mathbf{F}^{n, b}-\mathbf{f}^{n, b}\right) \phi_{i} \mathrm{~d} \Gamma_{b, k}, \quad 1 \leq i \leq N_{p}
$$


where $\mathbf{F}^{n, b}$ is the flux normal to the edge $b$ of element $k$, and $\mathbf{f}^{n, b}$ is a normal numerical flux through edge $b$ of element $k$, which is chosen as the exact upwind flux in this work. The solution is defined through the interpolation at nodes on an element,

$$
q(x, y) \approx \sum_{j=1}^{N_{p}} q_{j}\left(x_{j}, y_{j}\right) \phi_{j}(x, y),
$$

where $q$ is an element of the vector, $\mathbf{U}$, and $q_{j}$ is the value at node, $j$, on the element.

It is important that the interpolation nodes, $x_{j}, y_{j}$, are chosen such that the above interpolation is well-behaved. The reader may refer to Hesthaven and Warburton ${ }^{20}$ for possible choices of these nodes. Substituting Equation (5) into the strong formulation, and defining mass, stiffness, and edge matrices, the semi-discrete formulation becomes

$$
\frac{\mathrm{d} U^{k}}{\mathrm{~d} t}=\mathbf{M}_{k}^{-1}\left[\mathbf{M}_{k} S^{k}-\left(\mathbf{S}_{x}^{k} F^{k}+\mathbf{S}_{y}^{k} G^{k}\right)+\sum_{b=1}^{3} \mathbf{M}_{k}^{b}\left(F_{k}^{n, b}-f_{k}^{n, b}\right)\right],
$$

where the elements of $\mathbf{M}^{k}, \mathbf{S}_{x}^{k}, \mathbf{S}_{y}^{k}$, and $\mathbf{M}_{k}^{b}$ are defined as

$$
\begin{aligned}
\left(\mathbf{M}_{k}\right)_{i j}=J_{k} \int_{R_{k}} \phi_{i} \phi_{j} \mathrm{~d} R_{k}, & \left(\mathbf{M}_{k}^{b}\right)_{i j}=\frac{s_{b}}{J_{k}} \int_{\Gamma_{b}} \phi_{i} \phi_{j} \mathrm{~d} \Gamma_{b}, \\
\left(\mathbf{S}_{x}^{k}\right)_{i j}=J_{k} \int_{R_{k}} \frac{\partial \phi_{j}}{\partial x} \phi_{i} \mathrm{~d} R_{k}, & \left(\mathbf{S}_{y}^{k}\right)_{i j}=J_{k} \int_{R_{k}} \frac{\partial \phi_{j}}{\partial y} \phi_{i} \mathrm{~d} R_{k}
\end{aligned}
$$

where $R_{k}$ is a reference triangular element, $J_{k}$ is the transformation Jacobian between element $k$ and the reference element, and $s_{b}$ is the length of edge $b$. The above four matrices can be computed and stored once for the reference element and only the transformation Jacobian, $J_{k}$, must be stored for every element.

\section{Time integration scheme}

The specific choice of time integration scheme depends on the relative importance of temporal accuracy to computational cost. For aeroacoustics, low-storage Runge-Kutta time-marching schemes are a popular choice due to only requiring the storage of two solution levels and obtaining high-order accuracy for smooth problems. While more evaluations of the residual are required compared to standard Runge-Kutta schemes, the memory cost is greatly reduced. The low-storage scheme used in this work is the six stage, fourth order accurate, RK46-L scheme of Berland et. $a l^{21}$ which uses optimized Runge-Kutta coefficients. A low-storage Runge-Kutta scheme with two storage levels may be written as

$$
u^{n+1}=u^{n}+\sum_{i=1}^{N_{s}} \gamma_{i} \Delta t^{i} F^{i}(u)
$$

where $n$ is the time level, $i$ is the stage, $N_{s}$ is the number of stages, $\Delta t$ is the time step, and $F$ is the right hand side of Equation (6).

The time step is calculated for each element by the CFL-type criterion

$$
\Delta t=\mathrm{CFL} \frac{d_{\min } r}{v_{\max }},
$$

where $d_{\min }$ is the minimum distance between two nodes on the reference element, $r$ is the inscribed radius of the element, and $v_{\max }$ is the maximum wave-speed. The time step is calculated for every element and the

minimum over all elements is taken as the global time step to ensure stability. Therefore, the global time step is a function of the smallest element size and the highest order.

\section{Numerical dispersion analysis for an infinite periodic mesh}

Consider a patch of two triangular elements in an infinite, periodic mesh as shown in Figure 1. For each 


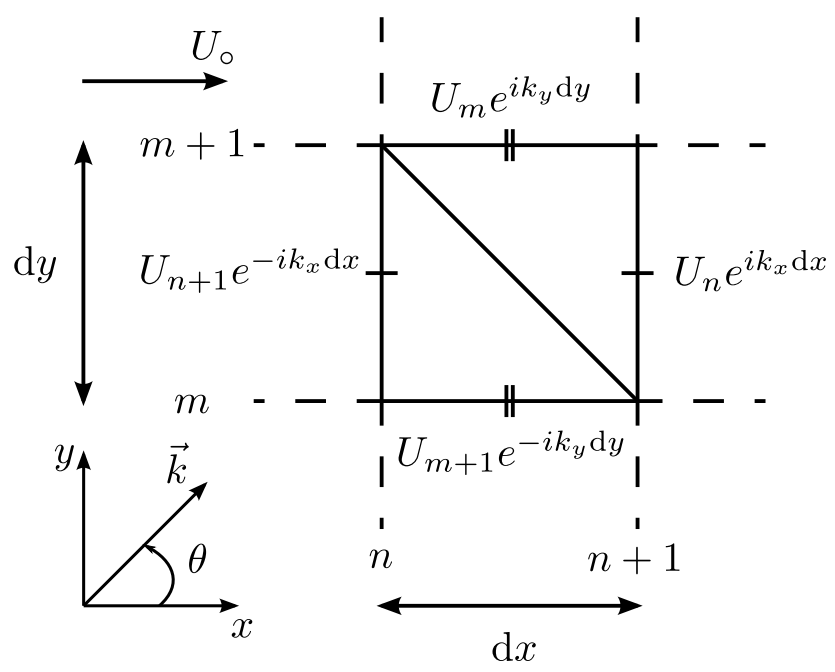

Figure 1: Formation of a dispersion analysis over an infinite, periodic mesh.

element, the degrees of freedom (DOF), which in this case are the solution values at the optimized nodes of the element, are stored as a vector,

$$
\mathbf{U}_{k}=\left[\rho_{1}, \rho u_{1}, \rho v_{1}, \pi_{1}, \ldots, \rho_{N_{p}}, \rho u_{N_{p}}, \rho v_{N_{p}}, \pi_{N_{p}}\right]^{\mathrm{T}},
$$

containing degrees of freedom at nodes $1,2, \ldots, N_{p}$ for each element, $k$. The degrees of freedom of both elements are concatenated into a single vector,

$$
\mathbf{U}=\left[\begin{array}{l}
\mathbf{U}_{1} \\
\mathbf{U}_{2}
\end{array}\right] .
$$

The surface integral in Equation (4) requires contributions from degrees of freedom on element edges and the application of periodic wave conditions on external edges of the patch. By using the definition of the upwind numerical flux and assuming periodicity of the solution at the external edges of the patch, the surface integral can be split into two terms,

$$
\sum_{b=1}^{3} \int_{\Gamma_{b, k}}\left(\mathbf{F}^{n, b}-\mathbf{f}^{n, b}\right) \phi_{i} \mathrm{~d} \Gamma_{b, k}=\left[\sum_{b=1}^{3} \mathbf{M}_{\Gamma_{k, b}}\left(\mathbf{F}_{n}-\mathbf{f}_{b}^{-}\right) \mathbf{T}_{b}^{-}+\sum_{b=1}^{3} \mathbf{M}_{\Gamma_{k, b}}\left(-\mathbf{f}_{b}^{+}\right) \mathbf{T}_{b}^{+}\right] \mathbf{U}=\boldsymbol{\Sigma}_{k}^{-}+\boldsymbol{\Sigma}_{k}^{+},
$$

separating the contributions to the integral from each element. The matrices, $\mathbf{T}_{b}^{ \pm}$are diagonal matrices containing ones at the indices corresponding to internal DOF of the element $k$ on edge $b\left(\mathbf{T}_{b}^{-}\right)$, and external DOF to the element $k$ on edge $b\left(\mathbf{T}_{b}^{+}\right)$and zeros elsewhere. To enforce periodic wave conditions on the patch, the $\mathbf{T}_{b}$ matrices for edges that are external to the patch are multiplied by $e^{ \pm i \vec{k} \cdot \hat{n}_{b}}$ using knowledge of the plane-wave periodicity. The matrices, $f_{b}^{+}$and $f_{b}^{-}$contain the operations required to compute the numerical flux using DOF exterior (+) to the face $b$, and interior $(-)$ to the face $b$, respectively. The term,

$$
\mathbf{F}_{n}=\mathbf{F} n_{x}+\mathbf{G} n_{y},
$$

is the flux normal to an element edge, evaluated using the DOF on the edge. The matrix, $\mathbf{M}_{\Gamma_{b}}$, is the mass matrix for edge $b$.

With the boundary integral term defined, the DGM system for the two-element patch can be assembled into

$$
\left[\begin{array}{cc}
\mathbf{M}_{1} & \mathbf{0} \\
\mathbf{0} & \mathbf{M}_{2}
\end{array}\right] \frac{\mathrm{d} \mathbf{U}}{\mathrm{dt}}+\left[\begin{array}{cc}
\mathbf{S}_{x_{1}} \mathbf{F}+\mathbf{S}_{y_{1}} \mathbf{G} & \mathbf{0} \\
\mathbf{0} & \mathbf{S}_{x_{2}} \mathbf{F}+\mathbf{S}_{y_{2}} \mathbf{G}
\end{array}\right] \mathbf{U}-\left[\begin{array}{cc}
\boldsymbol{\Sigma}_{1}^{-} & \boldsymbol{\Sigma}_{1}^{+} \\
\boldsymbol{\Sigma}_{2}^{+} & \boldsymbol{\Sigma}_{2}^{-}
\end{array}\right] \mathbf{U}=\mathbf{0},
$$

and may be manipulated to arrive at the equation for the residual,

$$
\frac{\mathrm{d} \mathbf{U}}{\mathrm{dt}}=\mathbf{M}^{-1}[\mathbf{S}-\mathbf{\Sigma}] \mathbf{U},
$$


which is the semi-discrete DGM formulation for the two element patch in Figure 1. To analyze the accuracy of the spatial DGM scheme alone, the time-derivative of the DOF vector can be replaced by the exact expression. Therefore solutions to the generalized eigenvalue problem,

$$
-i \tilde{\omega} \mathbf{U}=\mathbf{A} \mathbf{U}
$$

for the numerical frequencies, $\tilde{\omega}$, give the numerical dispersion relation, $D(\tilde{\omega}, k)$, of the semi-discrete scheme.

To analyze both the spatial and temporal accuracy of the scheme, the time-derivative of the solution can be discretized using the Runge-Kutta time-marching scheme defined in Section 1 resulting in

$$
e^{-i \tilde{\omega} \Delta t} \mathbf{U}=\left[\mathbf{I}+\sum_{s=1}^{N_{s}} \gamma_{s}(\mathbf{A} \Delta t)^{s}\right] \mathbf{U},
$$

where growth-rates, $e^{-i \tilde{\omega} \Delta t}$, of the solution between time steps $t^{n}$ and $t^{n+1}$ are found as eigenvalues. This results in a numerical dispersion relation dependent on the same parameters as in the semi-discrete case, but with the addition of a time step, $\Delta t$. Additionally, the stability can be analyzed by looking for modes with $e^{-i \tilde{\omega} \Delta t}>1$.

Solutions to Equation (16) or (17) provide $2 \times N_{p} \times N_{v}$ eigenvalues and corresponding eigenvectors. However, solutions of the continuous LEE are in the form of only acoustic, entropy, and vorticity modes. Therefore, most of the modes found by solving the eigenvalue problem are non-physical, spurious modes that are the result of discretizing the governing equations. To identify the physical modes, the numerical frequencies are compared to the exact frequencies determined by the dispersion relations for the acoustic, entropy, and vorticity modes.

\section{A. Error convergence study}

Using the two-element dispersion analysis method defined above, the spatial accuracy of the DGM with decreasing $k h$ is demonstrated in this section. The spectral accuracy of the DGM scheme is defined by how well the numerical frequencies, $\tilde{\omega}$, predict the exact frequencies governed by the exact dispersion relation of the LEE. Error in phase, called dispersion error, is caused by differences between the real part of the numerical frequency and the exact value. In this section, the dispersion error is reported as a relative dispersion error,

$$
E_{d}=\left|\frac{\omega-\operatorname{Re}(\tilde{\omega})}{\omega}\right|
$$

High dispersion error will result in a phase shift over long propagation distances. Error in the wave amplitude is caused by dissipation error, and is the result of the numerical frequency having a non-zero imaginary part. In this section, the dissipation error is reported as a non-dimensional dissipation error,

$$
E_{a}=\left|\frac{\operatorname{Im}(\tilde{\omega})}{\omega}\right|,
$$

that is, the ratio between the imaginary part of the numerical frequency and the exact frequency. High dissipation error will result in a large reduction in amplitude over long propagation distances.

The solution of the eigenvalue problem, Equation (16), for the eigenvectors gives the nodal solution values on the two-element patch. Eigenvectors corresponding to the acoustic, entropy, and vorticity modes can be found and compared to the exact solutions for each mode. This is done by computing the integrated $L^{2}$ error over the two-element patch for each mode,

$$
\|E\|_{2}=\int_{\Omega}\left|U_{e x}-\tilde{U}\right|^{2} \mathrm{~d} \Omega,
$$

where $\Omega$ is the two-element patch, $U_{e x}$ is the exact solution for the mode, and $\tilde{U}$ is the predicted solution for the mode. 


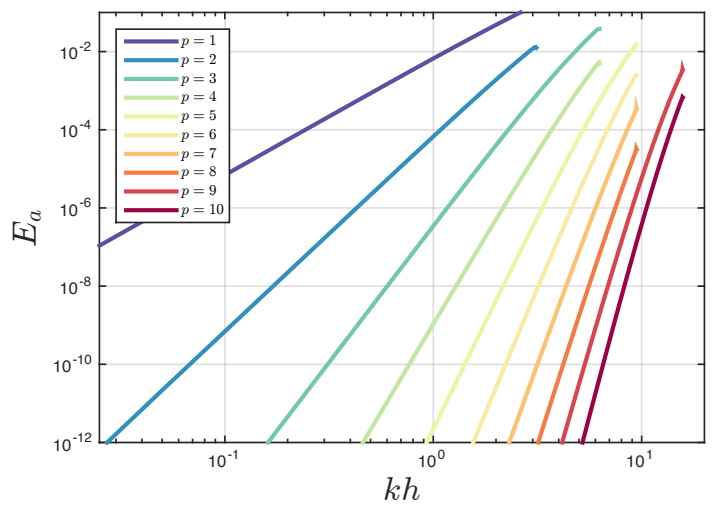

(a) Dissipation error vs. $k h$ for orders 1-10.

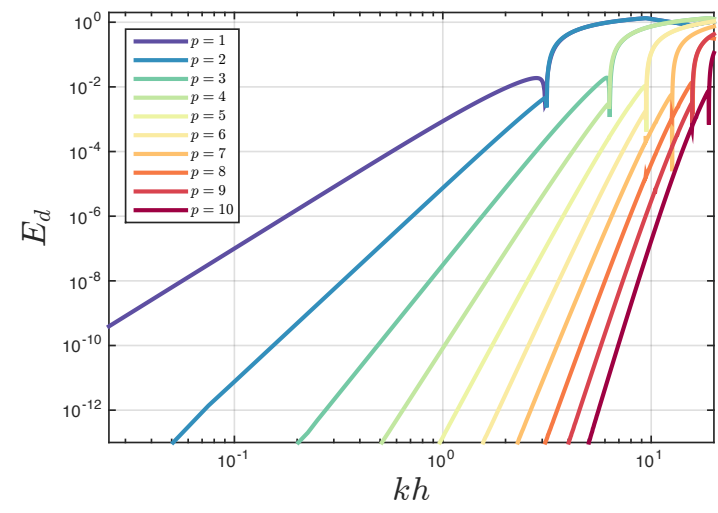

(b) Relative dispersion error vs. $k h$ for orders 1-10.

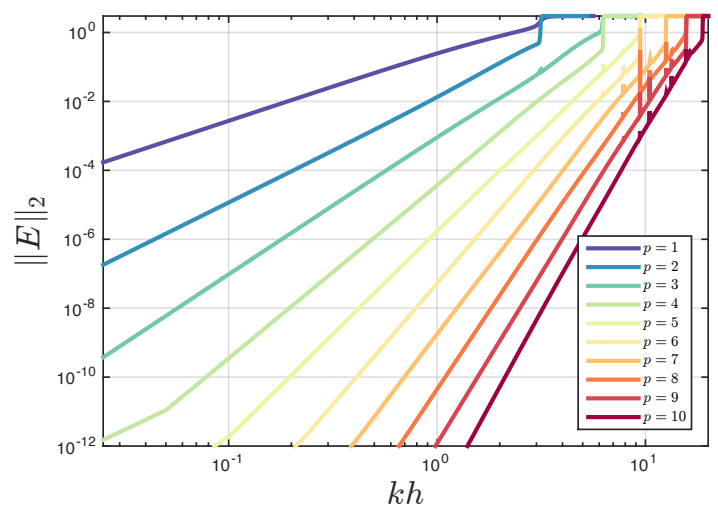

(c) Solution $L_{2}$ error vs. $k h$ for orders 1-10.

Figure 2: Semi-discrete convergence of the dissipation (a), relative dispersion (b), and integrated $L_{2}$ (c) errors with $k h$ for the acoustic mode. Each colored line represents convergence at a fixed polynomial order.

\section{Semi-discrete form}

The spatial accuracy of the DGM is evaluated by performing a convergence study of the errors defined above with $k h$. As mentioned in Section A, solutions of the LEE are made up of combinations of acoustic, entropy, and vorticity modes. These solution modes are also present in the numerical dispersion analysis, but with the addition of many spurious, non-physical modes caused by the numerical approximation of the governing equations. The accuracy associated with correctly predicting the acoustic mode with zero mean flow is studied first, followed by predictions of the acoustic, entropy, and vorticity modes in the presence of a mean flow.

To study the convergence of the acoustic mode, the element size, $h$, is fixed, and the wavenumber, $k$, is varied for polynomial orders, $1-10$. The direction of wave propagation is fixed in the positive $x$-direction $(\theta=0)$. Figures $2 \mathrm{a}-2 \mathrm{c}$ show the convergence behavior of the acoustic mode with zero mean flow velocity. At each polynomial order, a convergence rate can be extracted for each measure of error by computing the slope of the line. The dissipation, relative dispersion, and solution $L_{2}$ error convergence rates are found to be

$$
E_{a} \sim \mathcal{O}(k h)^{2 p+1}, \quad E_{d} \sim \mathcal{O}(k h)^{2 p+2}, \quad\|E\|_{2} \sim \mathcal{O}(k h)^{p+1},
$$

where $p$ is the polynomial order, and agree with theoretical predictions for the scalar advection equation. ${ }^{5}$

Error convergence of the entropy and vorticity modes can be compared to the acoustic mode by performing the same convergence study as above, but with a mean flow Mach number of $M=0.5$ in the positive $x$-direction. Figures 3a-3c show the convergence behavior of the acoustic, entropy, and vorticity modes with $k h$. Solid lines indicate the acoustic mode, while dashed and dot-dashed lines indicate the entropy and 


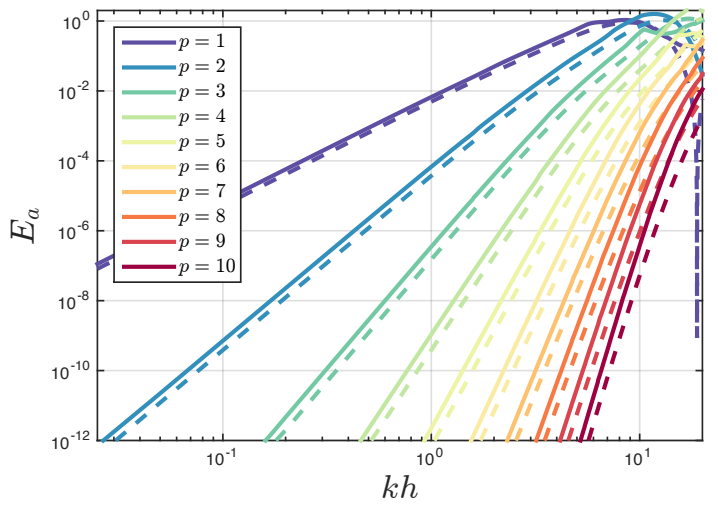

(a)

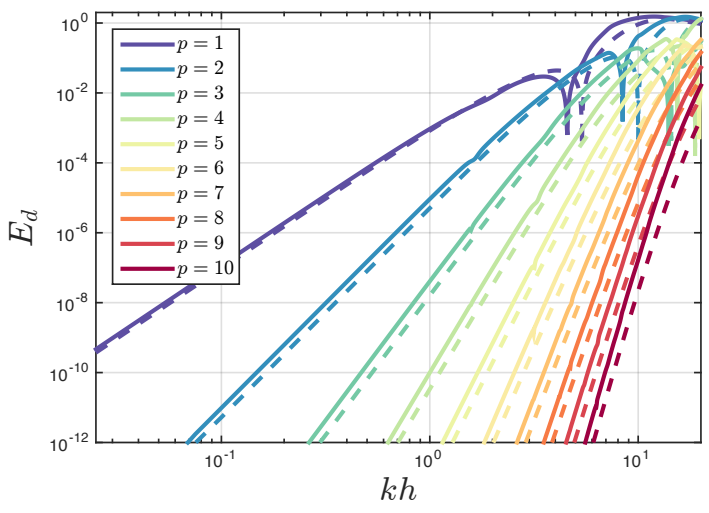

(b)

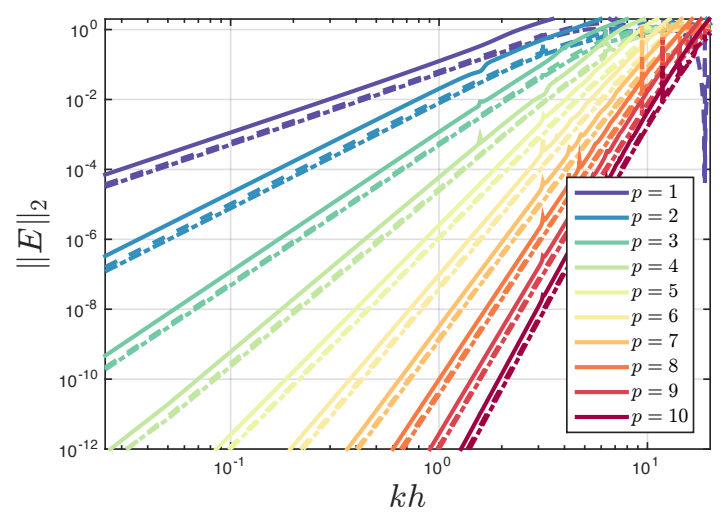

(c)

Figure 3: Semi-discrete convergence of the dissipation (a), relative dispersion (b), and integrated $L_{2}$ (c) errors with $k h$ for all modes. Solid lines indicate the acoustic mode, while dashed lines indicate the entropy and vorticity modes.

vorticity modes, respectively. Convergence rates of the individual modes agree with the rates found above for the zero mean flow problem for small $k h$. Since few differences can be seen between the convergence trends of the acoustic mode and the hydrodynamic modes, only the acoustic mode is analyzed in the following sections.

\section{Fully-discrete form}

Inclusion of time-discretization into the dispersion analysis shows how the temporal accuracy of the scheme impacts the optimal error convergence behavior demonstrated in the previous section. The time step, $\Delta t$, is bound by the stability requirements of the fully-discrete DGM and choices of this parameter impact both the stability and accuracy.

A grid-convergence study is performed by solving the fully-discrete eigenvalue problem defined by Equation (17). The wavenumber and propagation direction are fixed at $2 \pi$ and $\theta=0$, respectively, and the element size, $\Delta x$, is varied. The time step, $\Delta t$ is calculated by the CFL criterion in Equation (9). This additional parameter is accounted for by performing convergence studies for a range of CFL from 0.1 to 1.0. Again, the dissipation, dispersion, and integrated solution errors are found.

Figures 4a-4c show convergence of the dissipation, dispersion, and integrated solution errors with $k h$. Each colored line represents a particular choice of polynomial order. The solid and dashed lines show the convergence at different values of the CFL from 0.1 to 1.0. It is apparent that for large $k h$, the convergence behaves similarly to that of the semi-discrete case. For small $k h$, or highly spatially resolved waves, the dis- 


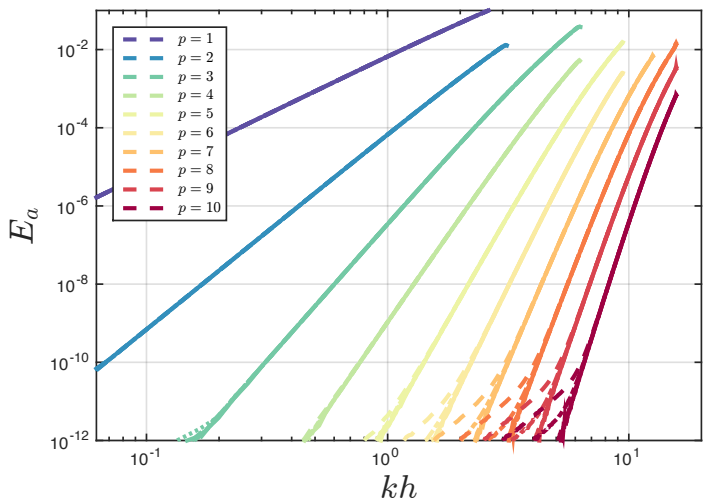

(a)

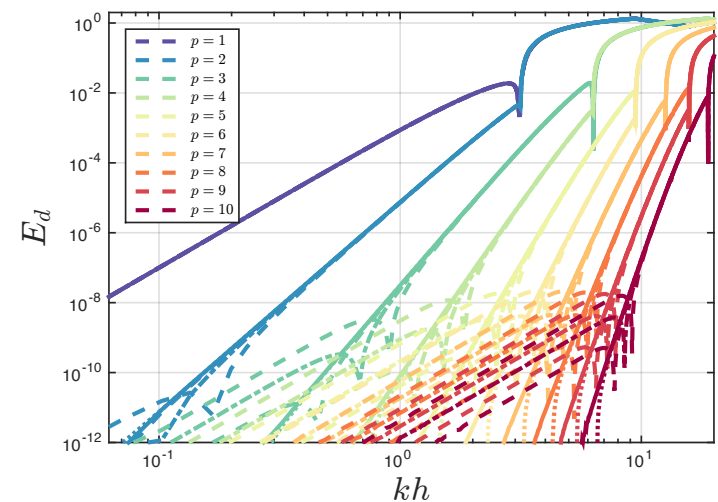

(b)

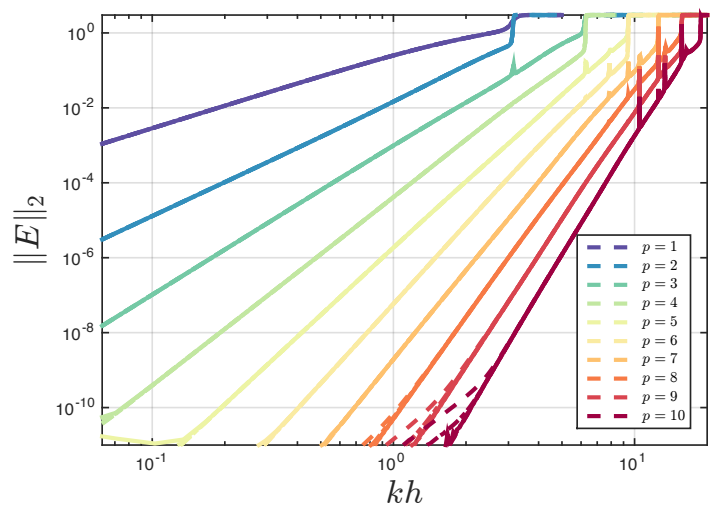

(c)

Figure 4: Dissipation, relative dispersion, and solution $L_{2}$ errors for orders 1-10 computed using the RK46-L scheme. Solid lines use a $\Delta t$ computed at a $\mathrm{CFL}=0.1$ and dashed lines show an increase in CFL.

persion error strongly deviates from the semi-discrete convergence rates, attaining a suboptimal convergence rate of $\mathcal{O}(k h)^{4}$. This fourth-order convergence rate is the order of accuracy of the time integration scheme. The dissipation error is only slightly impacted by the addition of temporal error, indicating that the time integration error is largely dispersive.

\section{B. Error anisotropy}

An important measure of accuracy for wave-propagation problems is the anisotropy, or how well the scheme can propagate waves in different directions relative to mesh or mean flow directions. While to estimate the impact of an arbitrary mesh or mean flow on the accuracy of the DGM would be impractical, this section serves to illustrate the general behavior of the accuracy with a small range of wave propagation directions and mean flows.

To isolate the purely mesh-induced anisotropy from the mean flow-induced anisotropy, the zero mean flow case is considered first. Referring to Figure 1, the direction of wave propagation is defined by the angle, $\theta$, between the $x$-axis and the wavenumber vector, $\vec{k}$. For $0 \leq \theta \leq 2 \pi$, and a range of wavenumbers, Figures $5 \mathrm{a}$ and $5 \mathrm{~b}$ show the relative dispersion error and the non-dimensional dissipation error. For $0 \leq \theta \leq 2 \pi$, and a range of polynomial orders at a fixed wavenumber, Figures $5 \mathrm{c}$ and $5 \mathrm{~d}$ show the relative dispersion error and the non-dimensional dissipation rate. As can be identified in the figures, there are preferred propagation directions through the two-element patch. Specifically, the propagation direction normal to the interface between elements is associated with the lowest error, while the direction tangential to the interface is associated with the highest error. Additionally, increasing the wavenumber and the polynomial order of 


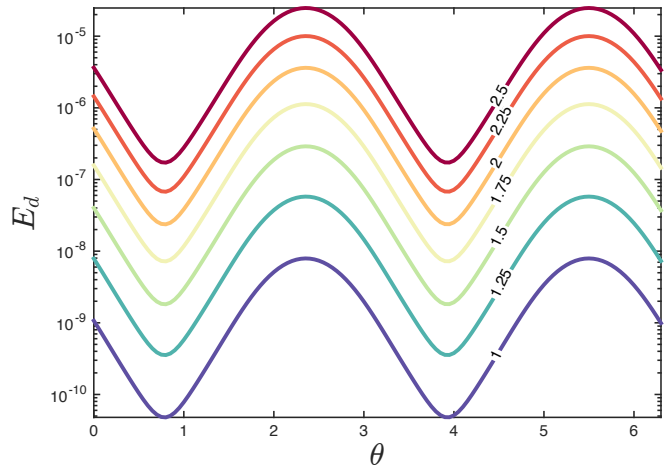

(a) Dispersion error for range of $\omega, M=0$

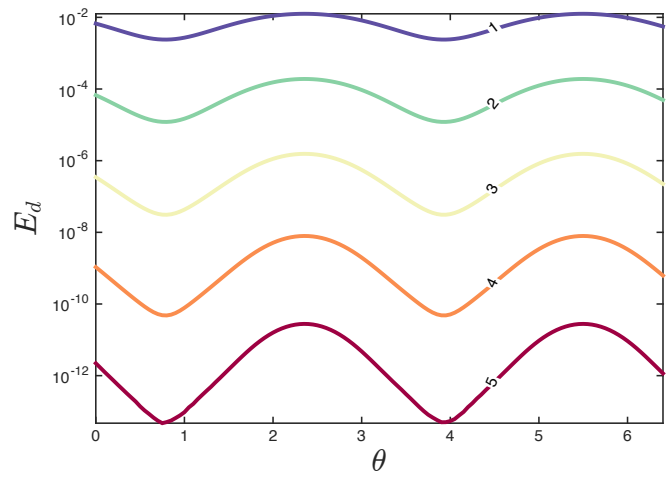

(c) Dispersion error for range of orders, $M=0$

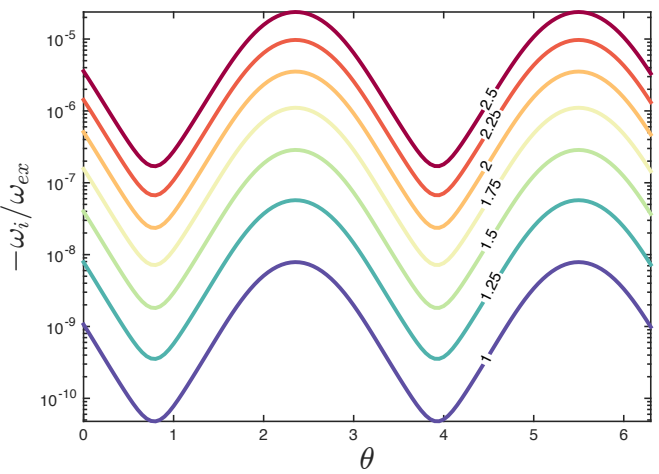

(b) Dissipation for range of $\omega, M=0$

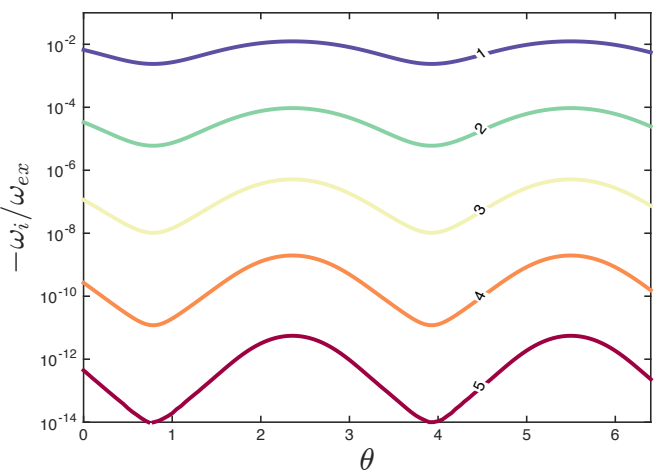

(d) Dissipation for range of orders, $M=0$

Figure 5: Anisotropic behavior of the relative dispersion error and the non-dimensional dissipation rate for a $0 \leq \theta \leq 2 \pi$ and zero mean flow. The top two figures show the effect of increasing frequency, $\omega$, and the bottom two figures show the effect of increasing the polynomial order, $p$, of the scheme at fixed frequency.

the scheme has the effect of increasing the anisotropy of the error.

To identify the anisotropy of the error induced by the mean flow, again, errors are calculated for $0 \leq \theta \leq$ $2 \pi$ in the presence of a mean flow with Mach number of $M=0.5$ in the positive $x$-direction. Rather than fix the wavenumber, the frequency is fixed and the relative dispersion error and non-dimensionalized dissipation error is calculated for the range of $\theta$. Figures $6 \mathrm{a}$ and $6 \mathrm{~b}$ show the behavior of the relative dispersion error and the non-dimensional dissipation rate for a range of frequencies. Figures $6 \mathrm{c}$ and $6 \mathrm{~d}$ show the behavior of the relative dispersion error and the non-dimensional dissipation error for a range of polynomial orders at a fixed frequency. The presence of a mean flow greatly impacts the anisotropic behavior of the error. The error is largest near the direction where the wave is propagating against the flow $(\theta=\pi)$. Propagation against the mean flow will have the effect of increasing the wavenumber, and therefore requiring more elements per wavelength to resolve the wave. Figures $6 \mathrm{a}-6 \mathrm{~d}$ show the maximum error occurring at a slightly shallower angle than the upstream direction, $\theta=\pi$. This can be attributed to competing errors, namely the error induced by the propagation direction in reference to the mean flow direction and the error induced by preferred propagation directions over the mesh itself.

\section{Parameter choices to control error and computational cost}

When solving realistic aeroacoustic problems, choosing element sizes, polynomial orders, and time steps, such that the solution is accurate, stable, and requires minimal computational cost, is not a straightforward task. This section seeks to provide insight into the choice of parameters for a wave propagation problem and their impact on both the accuracy and computational cost. The errors defined in Section 1 are re-defined in 


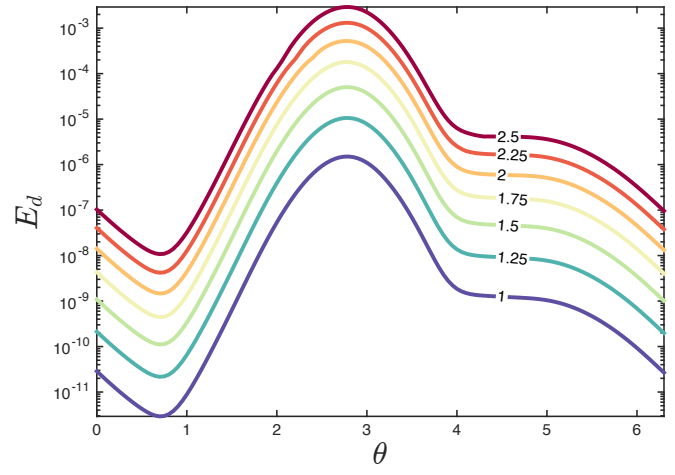

(a) Dispersion error for range of $\omega, M=0.5$

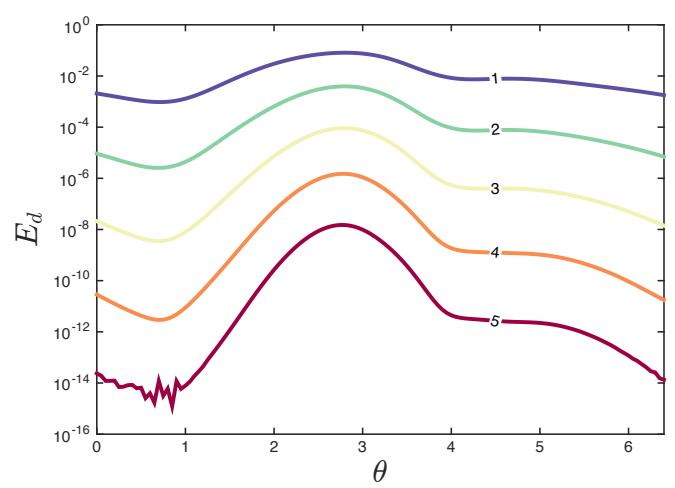

(c) Dispersion error for range of orders, $M=0.5$

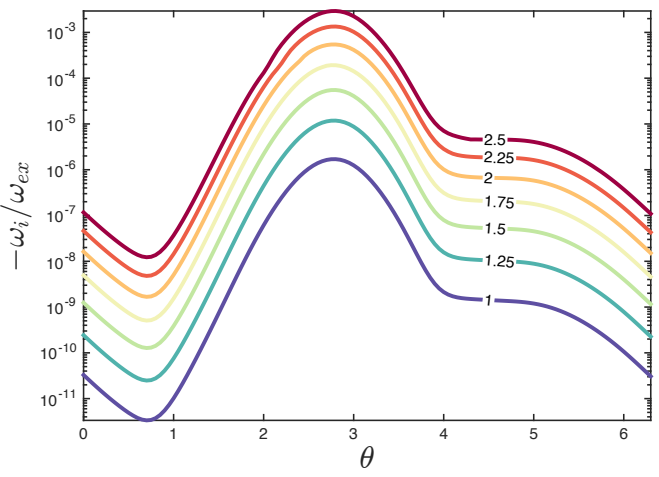

(b) Dissipation for range of $\omega, M=0.5$

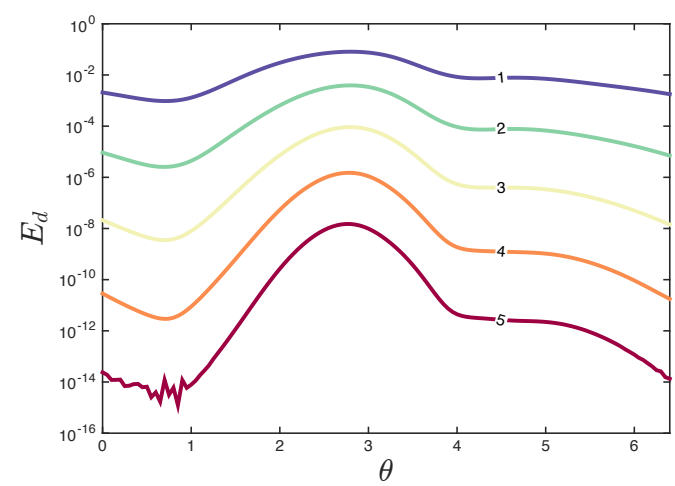

(d) Dissipation for range of orders, $M=0.5$

Figure 6: Anisotropic behavior of the relative dispersion error and the non-dimensional dissipation rate for a $0 \leq \theta \leq 2 \pi$ and mean flow of $M=0.5$. The top two figures show the effect of increasing frequency and the bottom two figures show the effect of increasing the polynomial order of the scheme at fixed frequency.

this section as the relative amplitude error,

$$
E_{a}=\left|1-e^{i \tilde{\omega}_{i} N_{T} T}\right|,
$$

and the relative position error,

$$
E_{d}=\left|1-\tilde{\omega}_{r} / \omega\right| N_{T},
$$

where $\tilde{\omega}_{i}$ and $\tilde{\omega}_{r}$ are the imaginary and real parts of the numerical frequency, $T$ is the period of oscillation, and $N_{T}$ is the number of periods. These errors can be thought of as the percentage reduction in amplitude due to dissipation and in position due to dispersion error. Since dispersion and dissipation errors build up in the solution as the waves propagate, it is important to also understand what impact the choice of polynomial order, mesh size, and time step have on the errors for both short and long propagation distances. First, optimal discretizations in terms of mesh size and order are found for short and long propagation distances. Second, the computational cost of the optimal discretizations is estimated and then used to develop guidelines for the choice of parameters for minimizing the computational cost.

\section{Accuracy for short and long propagation distances}

Accuracy is computed for short and long distance propagation by performing a mesh convergence study for orders $1-10$ and calculating the error from Equations (22) and (23). For these convergence studies, the CFL is fixed to 1. First, accuracy is analyzed for a short distance propagation of one period of oscillation. Figures $7 \mathrm{a}$ and $7 \mathrm{c}$ show the convergence of the relative amplitude and position error with the number of elements per squared wavelength, $N_{e} / \lambda^{2}$. From the convergence plots, optimal discretizations of order and 


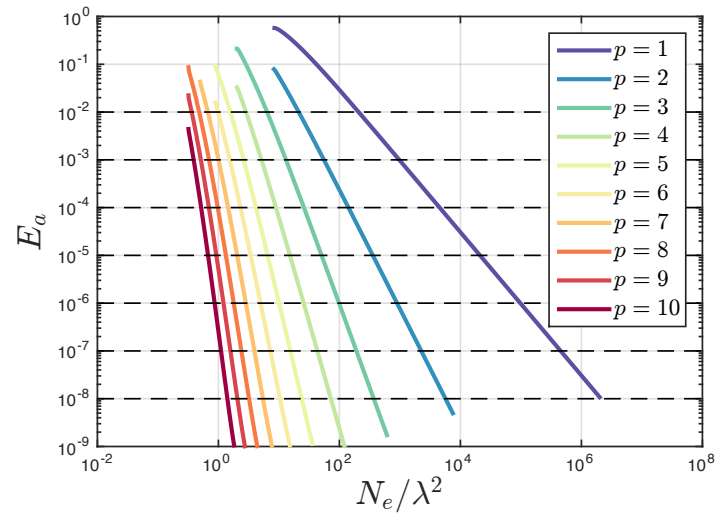

(a)

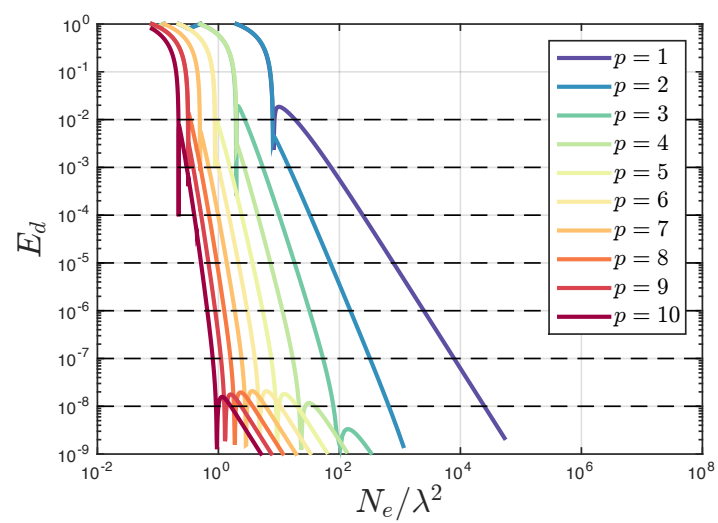

(c)

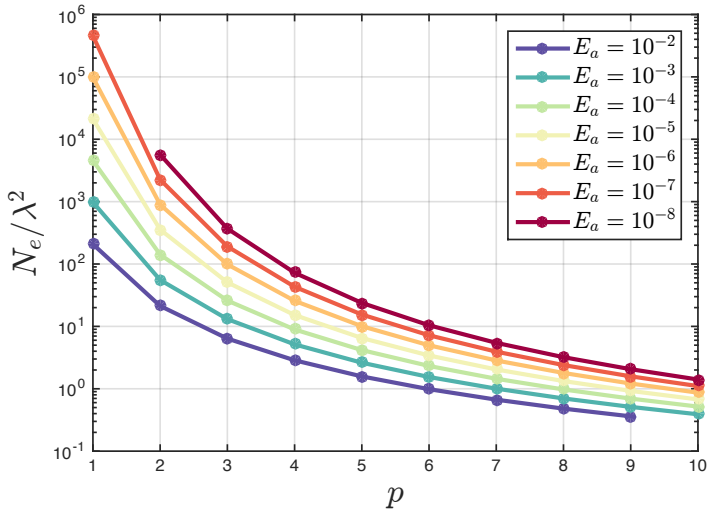

(b)

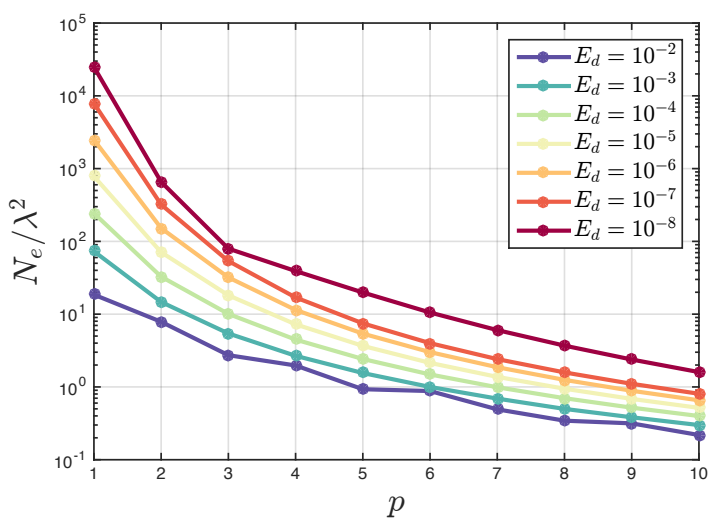

(d)

Figure 7: Convergence of $E_{a}(\mathrm{a})$ and $E_{d}(\mathrm{c})$ and optimal discretizations to achieve fixed $E_{a}(\mathrm{~b})$ and $E_{d}(\mathrm{~d})$ for one period of oscillation.

mesh size are selected for each order at the fixed accuracy levels dictated by the dashed lines. The optimal discretizations are then compared in Figures $7 \mathrm{~b}$ and $7 \mathrm{~d}$ at fixed amplitude and position error, respectively. For the amplitude error optimal discretizations, it is clear that at all accuracy levels, increasing the order affords a sharp reduction in the number of elements per wavelength. This is also true for the position error, except at the highest accuracy level, $E_{d}=10^{-8}$, where the temporal error convergence rate of $\mathcal{O}\left(\Delta t^{4}\right)$, dominates. Next, the same analysis is applied to a long distance propagation of 100 periods of oscillation. Figures $8 \mathrm{a}$ and $8 \mathrm{~b}$ show the amplitude reduction error. Again, for the amplitude reduction error, increasing order results in a sharp decrease in the required number of elements per squared wavelength to achieve a particular accuracy level. Figures $8 \mathrm{c}$ and $8 \mathrm{~d}$ show the same analysis applied to the position error. Increasing the propagation distance effectively increases the impact of the temporal error for high accuracy levels. Note that for low accuracy levels, the number of elements required to achieve a particular position accuracy is less than the number required for the amplitude error. However, at high accuracy levels and/or long propagation distances, the position error places stricter requirements on element size. For engineering levels of accuracy, e.g. $E_{a}=E_{d}=10^{-3}$, the spatial error dominates the temporal error.

\section{Computational cost}

To roughly estimate the computational cost, every floating point operation required to compute the spatial operator and integrate the equations in time, is identified. This estimated cost can be computed by

$$
C=n_{t} n_{s} n_{e}\left(2 M_{\mathrm{div}}+n_{f} M_{F n}+T+S_{F}+F+F_{\partial}\right),
$$




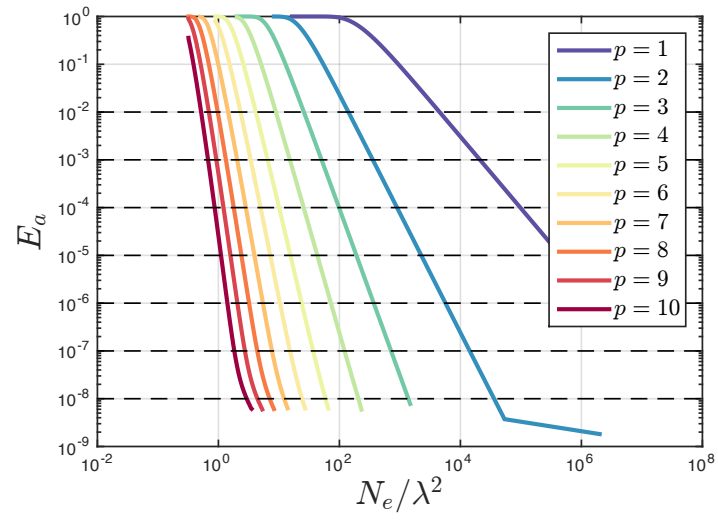

(a)

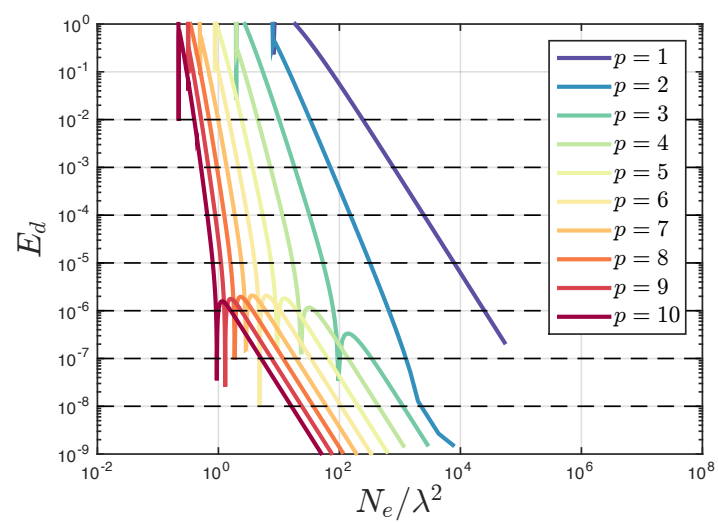

(c)

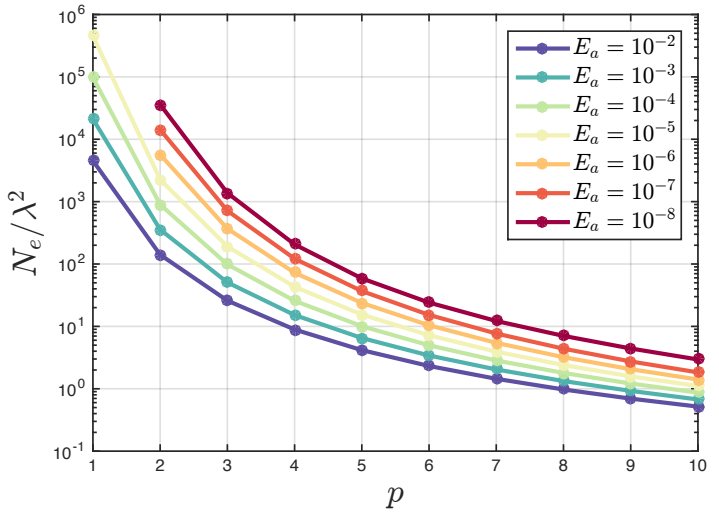

(b)

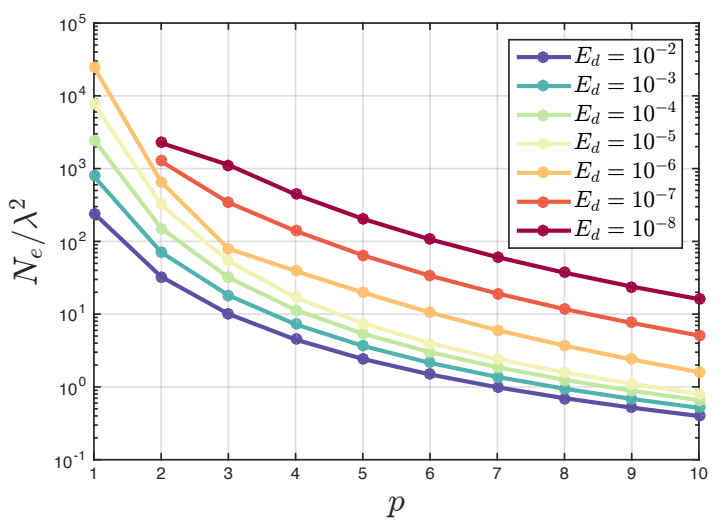

(d)

Figure 8: Convergence of $E_{a}(\mathrm{a})$ and $E_{d}(\mathrm{c})$ and optimal discretizations to achieve fixed $E_{a}(\mathrm{~b})$ and $E_{d}$ (d) for 100 periods of oscillation.

where $M_{\text {div }}$ and $M_{F n}$ are the operations associated with matrix multiplications for the divergence of the flux and the boundary flux, $T$ is the time integration, $S_{F}$ is the scaling of the flux in the quadrature-free formulation, $F$ is the computation of the interior fluxes, and $F_{\partial}$ is the computation of the element boundary fluxes. The variables, $n_{f}, n_{t}$, and $n_{s}$ are the number of element faces, number of time steps, and the number of time integration stages, respectively. In addition to this estimation, there will be important overheads associated with the communication costs between processors, the memory usage, and other architecture specific costs, but these effects are not directly accounted for in this analysis.

Similar to the error analysis above, the computational cost is computed for fixed amplitude and position accuracy levels at both short and long propagation distances. Figures $9 \mathrm{a}$ and $9 \mathrm{c}$ show the computational cost required to achieve fixed levels of amplitude and position accuracy using a CFL $=1.0$. Each point along the curves corresponds to a particular optimal discretization of $N_{e} / \lambda^{2}$ and order, $p$. For a short propagation distance, Figure 9a indicates that the cost required to achieve a particular amplitude error level is higher than the cost required to achieve a particular position error. This can be seen by noticing that at each error level, the amplitude error curve (dashed line) is above or directly on top of the position error curve (solid line). At low accuracy levels, marginal cost reduction is achieved for high orders. The same analysis is repeated using a $\mathrm{CFL}=0.5$ in Figure 9b. It is apparent that reducing the CFL reduces the temporal error, but increases the cost. Therefore at short propagation distances and all but the highest accuracy levels, it is beneficial in terms of cost to maximize the CFL.

Keeping the CFL fixed to 1 and increasing the propagation distance, the cost analysis is repeated in Figure 9c. For lower accuracy levels, $E_{a}=E_{d}=10^{-2}$ to $E_{a}=E_{d}=10^{-6}$, amplitude error dictates the computational cost. In this case, at low accuracy levels, using high orders results in a larger relative cost 


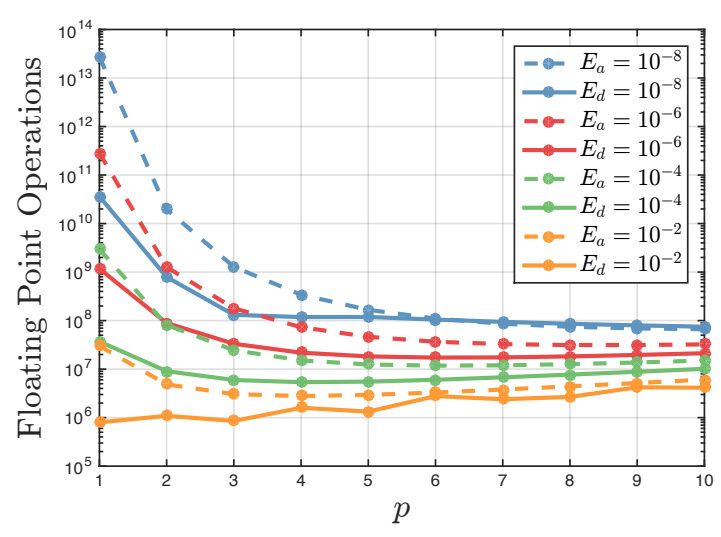

(a)

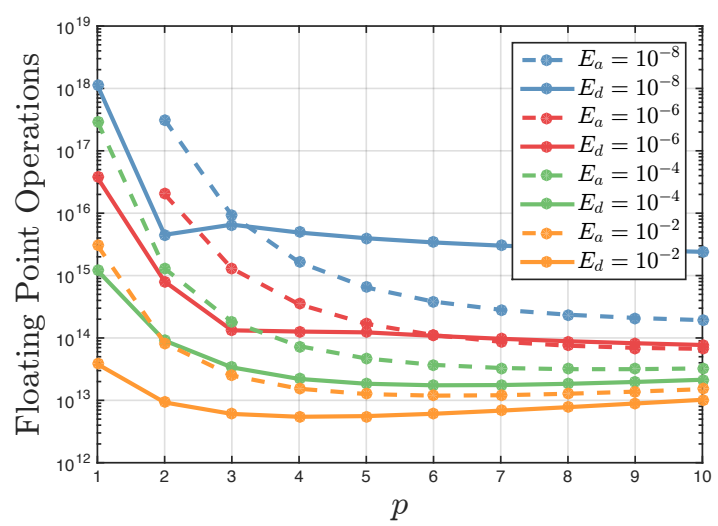

(c)

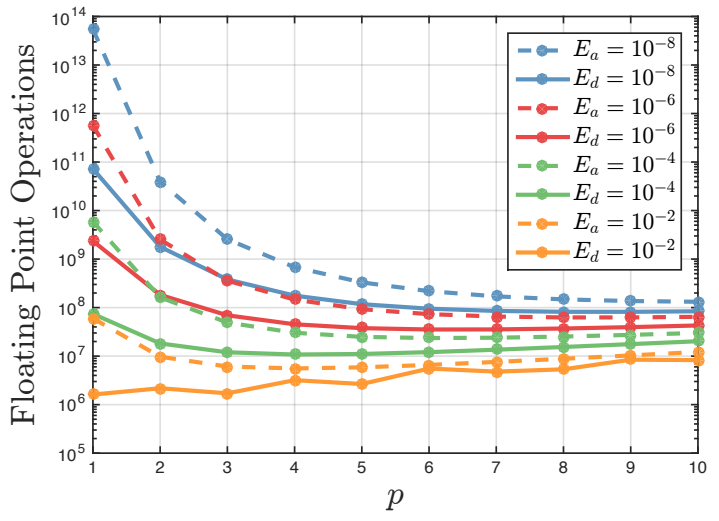

(b)

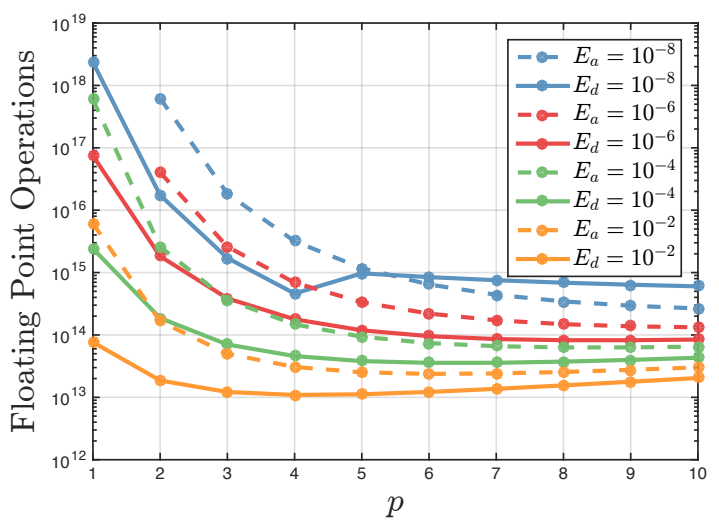

(d)

Figure 9: Estimated computational cost for fixed accuracies using a CFL = 1.0 (a) and a CFL $=0.5$ (b) for one period of oscillation. Estimated computational cost for fixed accuracies using a CFL = 1.0 (c) and a $\mathrm{CFL}=0.5(\mathrm{~d})$ for 100 periods of oscillation

reduction than in the short propagation case. However, at $E_{a}=E_{d}=10^{-8}$, the position error clearly dominates. This error level can be identified in Figure 8c as the temporal error dominated region. Note that increasing the order has very little impact on cost reduction when the error is temporally dominated. The same analysis is repeated using a CFL $=0.5$ in Figure 9d. For low accuracy levels, decreasing the CFL increases the cost. However, for the highest accuracy level, decreasing the CFL results in a reduction in cost. Decreasing the CFL reduces the temporal error, allowing the use of larger higher order elements. These results are summarized in Figure 10. Therefore, it may be logical to choose the element size and polynomial order to minimize the amplitude error, then determine which regime of Figure 10 is applicable to the problem. If for instance low accuracy and long propagation distance are required, the CFL should be maximized. If high accuracy and a long propagation distance is required, the CFL should be reduced to limit the temporal component of the position error. It is important to note that these results depend on the accuracy and the computational cost of the time integration scheme. For a different choice of time integration scheme than the one used in this work, the results may be different. However, these results may still be used as guidelines since only qualitative conclusions are made.

\section{Dispersion analysis for a semi-infinite channel}

A dispersion analysis performed for an infinite mesh, such as in Section III, is a useful tool for understanding the accuracy and stability of the scheme for uniform flow. However, most aeroacoustics problems 


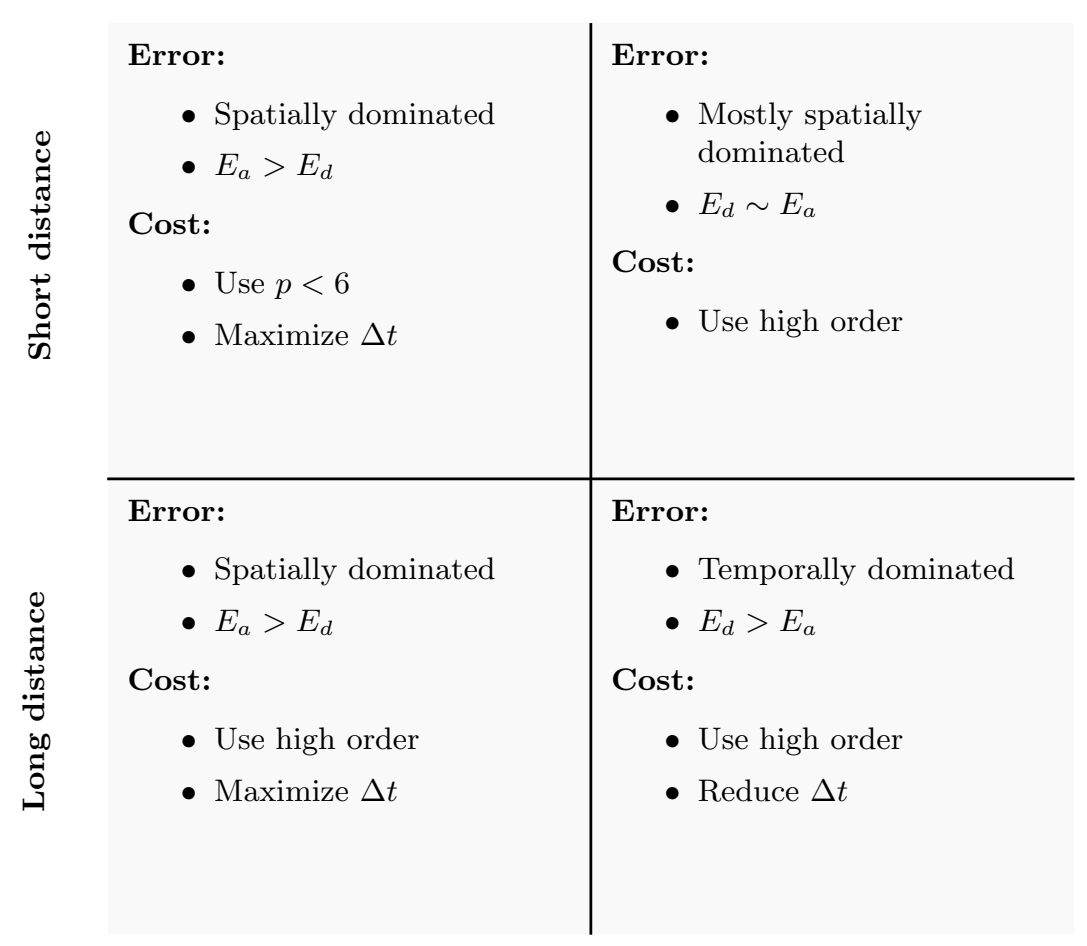

Figure 10: Guidelines for parameter choices based on low/high accuracy requirements and short/long distance propagation.

of interest involve highly non-uniform mean flows that support the formation of hydrodynamic instabilities. Additionally, numerical issues such as the accuracy of mean flow representation and aliasing error are important sources of error that cannot be captured by a more traditional dispersion analysis. Therefore, having a dispersion analysis technique that supports these mean flows may be an important tool to assess the performance of the scheme.

This section develops a technique for a fully-discrete dispersion analysis of the LEE which supports a non-uniform mean flow and uses it to study the formation of instabilities. This approach is also valid for the analysis of other features including the testing of boundary conditions, such as acoustic liner models or perfectly matched layers, or even the testing of various mean flow interpolation strategies. Gabard and Brambley developed a similar dispersion analysis for a finite-difference scheme and used it to study the Myers impedance boundary condition. ${ }^{22}$

Analogous to the above two-element dispersion analysis, a dispersion analysis of a semi-infinite channel problem can be constructed. Consider the periodic mesh in a hard-walled channel shown in Figure 11a. Instead of assuming a fixed size patch of elements with periodic wave conditions on all sides, periodicity is imposed only in the axial direction of the channel, $x$, and the transverse direction, $y$, is fully discretized, including boundary conditions at the walls. In contrast to the two-element case, no assumptions are made about the mean flow in the transverse direction, allowing one to prescribe a fully non-uniform mean flow.

Proceeding in a similar manner to the 2-element problem, a vector containing all DOF can be defined by simply concatenating each element's DOF vector to get

$$
\mathbf{U}=\left[\begin{array}{c}
\mathbf{U}_{1} \\
\mathbf{U}_{2} \\
\vdots \\
\mathbf{U}_{N_{k}}
\end{array}\right],
$$




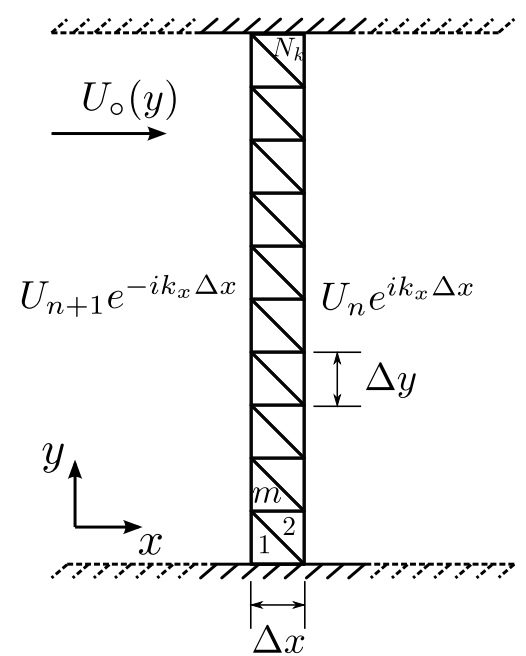

(a)

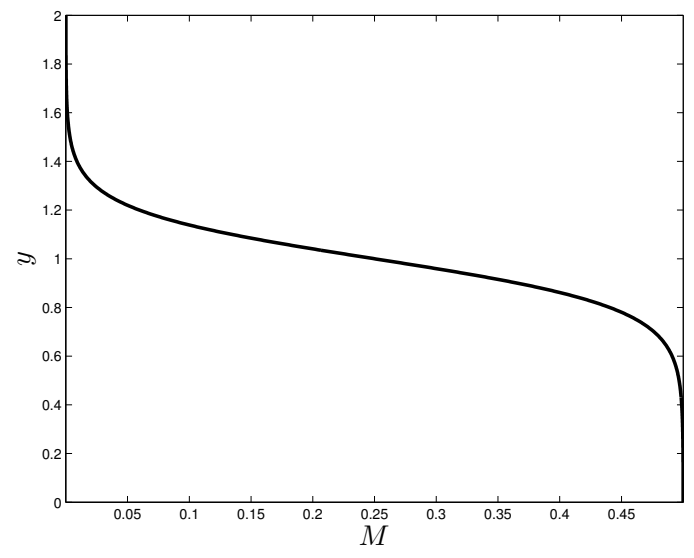

(b)

Figure 11: Construction of a fully-discrete dispersion analysis for a channel flow (a). Mean flow $x$-direction Mach number profile in a channel (b).

where $N_{k}$ is the total number of elements in the channel, which can take any even integer value. The ordering of DOF is defined based on the numbering strategy shown in Figure 11a to make edge flux computations straightforward.

As the channel mesh is a repeated pattern of two-element patches, the problem can be assembled in a similar way to the above analysis for two elements. For element patches that do not contain edges touching the walls, the surface integral term, Equation (12), can be used with only small modifications. The matrix, $\mathbf{T}_{b}^{ \pm}$now only enforces wave periodicity in the axial direction, and the boundary flux matrices, $\boldsymbol{\Sigma}_{k}^{ \pm}$, contain contributions to the flux from DOF internal to the patch, $(-)$, and external to the patch, $(+)$, e.g. patches $m-1$ and $m+1$. Assembling all patches of elements into a linear system results in the semi-discrete equation,

$$
\frac{\mathrm{d} \mathbf{U}}{\mathrm{dt}}=\mathbf{M}^{-1}\left[\begin{array}{ccccc}
\mathbf{E}_{1,2} & \boldsymbol{\Sigma}_{3}^{p+1} & \mathbf{0} & \ldots & \mathbf{0} \\
\boldsymbol{\Sigma}_{2}^{p-1} & \mathbf{E}_{3,4} & \boldsymbol{\Sigma}_{5}^{p+1} & \ldots & \mathbf{0} \\
\vdots & & \ddots & & \vdots \\
\mathbf{0} & \ldots & \mathbf{0} & \boldsymbol{\Sigma}_{N_{k}-2}^{p-1} & \mathbf{E}_{\left(N_{k}-1\right), N_{k}}
\end{array}\right] \mathbf{U}
$$

which is a block-tridiagonal matrix. The mass matrix, $\mathbf{M}$ is a block-diagonal matrix, containing each element's mass matrix. The flux terms corresponding to DOF on patch, $p=n, n+1$, are defined as

$$
\mathbf{E}_{n-1, n}=\left[\begin{array}{cc}
\mathbf{S}_{x_{n-1}} \mathbf{F}+\mathbf{S}_{y_{n-1}} \mathbf{G} & \mathbf{0} \\
\mathbf{0} & \mathbf{S}_{x_{n}} \mathbf{F}+\mathbf{S}_{y_{n}} \mathbf{G}
\end{array}\right] \mathbf{U}-\left[\begin{array}{cc}
\boldsymbol{\Sigma}_{n-1}^{-} & \boldsymbol{\Sigma}_{n-1}^{+} \\
\boldsymbol{\Sigma}_{n}^{+} & \boldsymbol{\Sigma}_{n}^{-}
\end{array}\right],
$$

and the contribution to the edge flux terms from DOF outside of patch $p$ are

$$
\boldsymbol{\Sigma}_{n}^{p-1}=\left[\begin{array}{cc}
\mathbf{0} & \boldsymbol{\Sigma}_{n-1}^{+} \\
\mathbf{0} & \mathbf{0}
\end{array}\right], \quad \boldsymbol{\Sigma}_{n}^{p+1}=\left[\begin{array}{cc}
\mathbf{0} & \mathbf{0} \\
\boldsymbol{\Sigma}_{n}^{+} & \mathbf{0}
\end{array}\right] .
$$

Proceeding in the same way as in the two-element case, the time-derivative of the DOF, $\frac{\mathrm{dU}}{\mathrm{dt}}$, can be either analytically prescribed, based on the known plane-wave solution, or discretized in time using a time integration scheme such as RK46-L. ${ }^{21}$

\section{A. Numerical instabilities in a sheared mean flow}

Shear layers are a common feature of turbofan exhaust flows, forming at the interface of the bypass and the free stream, and the core and bypass stream. Acoustic radiation from the core and bypass ducts interacts 
with the sheared flow, causing refraction of the waves. Modeling this physical feature is therefore crucial for the accurate prediction of far-field noise.

Solutions to the LEE in the presence of a sheared mean flow can permit the formation of hydrodynamic instabilities. For a range of frequencies, the unstable modes can grow undamped due to the lack of a dissipation mechanism in the governing equations. The amplitude of these modes can quickly grow, and distinguishing the acoustic solution from the instability can become difficult. Damping these modes is not the focus of this work, rather this section uses the unstable modes to understand the accuracy and stability of the numerical scheme.

To model the shear layer, a hyperbolic tangent axial Mach number profile,

$$
M=\frac{1}{4}\left(1+\tanh \left[\frac{H}{2 d}\left(1-\frac{|y|}{H}\right)\right]\right),
$$

where $H$ is the location of the inflection point and $d$ is the width, is imposed on the channel. The profile is illustrated in Figure 11b. The velocity is directly imposed on the DGM nodes, meaning that for an increase in polynomial order, the interpolation accuracy of the mean flow improves. For this study, a mesh with $N_{e}=20$ elements along the transverse direction of the channel is used.

For a range of axial wavenumbers and polynomial orders, the solution to the eigenvalue problem, Equation (26), is found and the eigenvalues associated with the most unstable modes, i.e. $\max (+\operatorname{I} m(\tilde{\omega}))$, are stored. Figure 12a shows the growth rates of the most unstable modes. It is clear that with increasing polynomial order, the growth rates do not converge to a single profile and that growth rates increase over a wide range of wavenumbers. This lack of convergence with order indicates that the instability is numerical, rather than a feature of the governing equations. Figure 12b shows the real part of the numerical frequency of the most unstable modes for each order. It is possible to determine whether the instability convects through the domain (convective instability) or is stationary (absolute instability) by calculating the group velocity, $\operatorname{Re}\left(\frac{\mathrm{d} \omega}{\mathrm{dk}}\right)$, from Figure 12b. The black, dashed line corresponds to the physical instability with a group velocity of $\operatorname{Re}\left(\frac{\mathrm{d} \omega}{\mathrm{dk}}\right)=0.25$, which is the centerline velocity in the shear layer. For the orders, $p=3$ to $p=5$, there is a range of wavenumbers where the numerical solution accurately predicts the group velocity of the physical mode. For higher orders, the group velocity clearly does not agree with the physical unstable mode and is nearly zero for the range of wavenumbers considered. This indicates that the numerical instability will not convect out of the domain.

This type of numerical instability is likely caused by aliasing error, which is a consequence of representing the flux in Equation (4) by interpolation at the same order as the solution, $p$. Since the flux is a product of mean flow terms and the solution, it is effectively a polynomial of order greater than $p$ and energy from higher order modes is transferred to the lower order modes in the solution, leading to an instability. ${ }^{20}$

A possible solution to the aliasing problem could be to apply a filter to the modal coefficients of the solution to damp the buildup of high-order oscillations, but care must be taken to add the minimum required

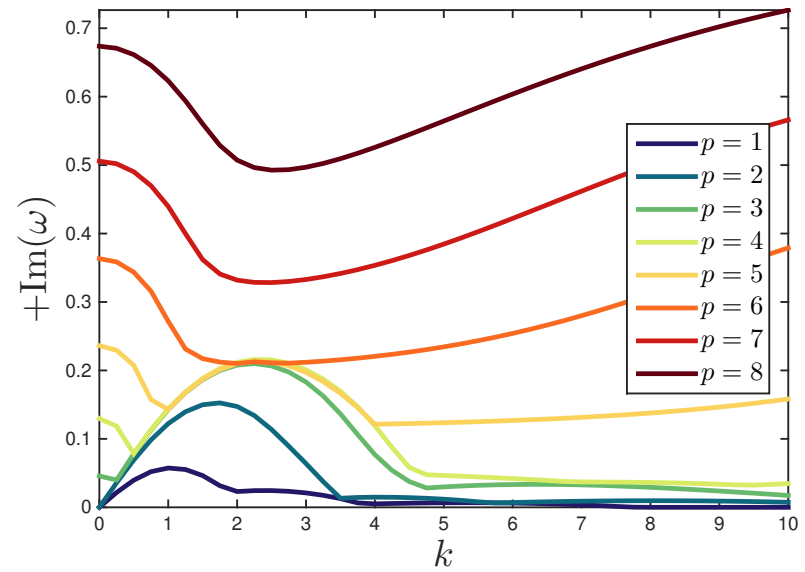

(a)

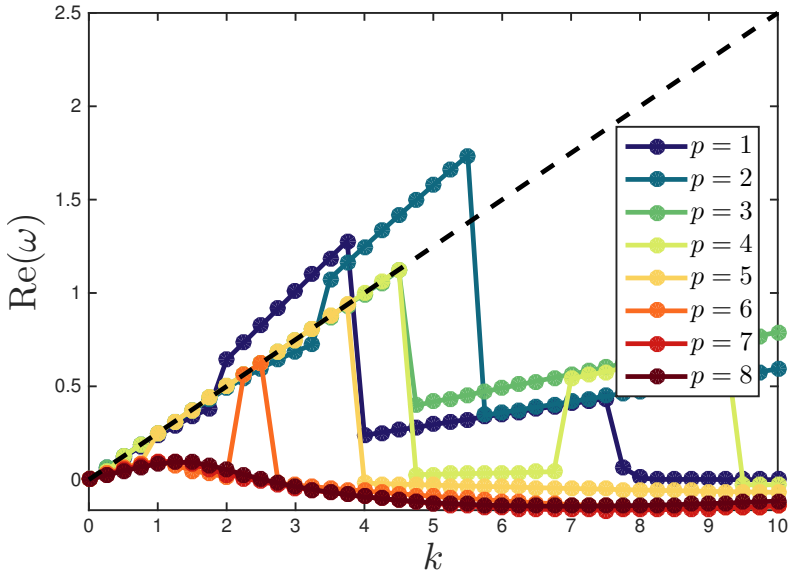

(b)

Figure 12: Growth rates of the most unstable mode for a range of orders. 
dissipation to the scheme so as to not damp out physical parts of the solution. ${ }^{20}$ Another solution could be to compute the flux on the $2 p$-order basis, then project onto the $p$-order basis, however the computational cost would be excessive. ${ }^{20}$ This dispersion analysis will be used in future work to develop an efficient and accurate solution to the aliasing problem.

\section{Conclusion}

The performance of the DGM for the solution of the linearized Euler equations has been demonstrated by using a two-dimensional dispersion analysis technique. In contrast to a typical dispersion analysis, no model equation has been used, and therefore it contains the full form of the LEE. Spectral accuracy of the scheme compares well with theoretical estimates for acoustic, entropy, and vorticity modes. Anisotropy of the error has been studied for acoustic propagation over a uniform mesh and varied mean flow directions. Guidelines for the choice of problem-specific, simulation parameters have been given and contrasted to computational cost estimates of the scheme.

The results indicate that for low to medium accuracy requirements, or short wave propagation distance, dissipation is the dominant source of error, caused by a lack of spatial resolution. For high accuracy requirements and long distance propagation, dispersion error is the dominant source of error, due to the fixed, $\mathcal{O}\left(\Delta t^{4}\right)$ accuracy of the time integration scheme used in this work. Computational cost estimates, in terms of total floating point operations, indicate that for high accuracy and long distance propagation, reducing the time step may be required to recover the computational cost benefits of a high order discretization.

A new dispersion analysis technique for analyzing the DGM-discretized LEE in the presence of a nonuniform mean flow has been proposed. This technique has been applied to study the behavior of instabilities in a sheared flow. Aliasing-like errors have been found to significantly contribute to the formation of unphysical, unstable modes in non-uniform mean flow regardless of the size of the polynomial basis used, and indicate that control of these modes is necessary to achieve a stable and accurate solution. Future work will use the dispersion analysis developed in Section IV to further understand the behavior of aliasing instabilities for highly non-uniform mean flows. Solutions to the aliasing problem that minimally impact the accuracy and computational cost of the scheme will be investigated.

\section{Acknowledgments}

This work is performed as part of the CRANE (Community and Ramp Aircraft NoisE) project and is supported in full by European Union funding under the Framework Programme 7. 


\section{References}

${ }^{1}$ H. L. Atkins and C.-W. Shu, "Quadrature-Free Implementation Of The Discontinuous Galerkin Method For Hyperbolic Equations," Tech. Rep. 96-51, NASA Langley Research Center, Hampton, Virginia, Aug. 1996.

${ }^{2}$ F. Q. Hu, M. Hussaini, and P. Rasetarinera, "An Analysis of the Discontinuous Galerkin Method for Wave Propagation Problems," Journal of Computational Physics, vol. 151, pp. 921-946, May 1999.

${ }^{3}$ F. Q. Hu and H. L. Atkins, "Eigensolution Analysis of the Discontinuous Galerkin Method with Nonuniform Grids," Journal of Computational Physics, vol. 182, pp. 516-545, Nov. 2002.

${ }^{4}$ M. Ainsworth, P. Monk, and W. Muniz, "Dispersive and Dissipative Properties of Discontinuous Galerkin Finite Element Methods for the Second-Order Wave Equation," Journal of Scientific Computing, vol. 27, pp. 5-40, June 2006.

${ }^{5} \mathrm{M}$. Ainsworth, "Dispersive and dissipative behaviour of high order discontinuous Galerkin finite element methods," Journal of Computational Physics, vol. 198, pp. 106-130, July 2004.

${ }^{6}$ J. Alvarez, L. D. Angulo, M. R. Cabello, A. R. Bretones, and S. G. Garcia, "An Analysis of the Leap-Frog Discontinuous Galerkin Method for Maxwell's Equations," IEEE Transactions on Microwave Theory and Techniques, vol. 62, pp. 197-207, Feb. 2014.

${ }^{7}$ N. Chevaugeon, K. Hillewaert, X. Gallez, P. Ploumhans, and J.-F. Remacle, "Optimal numerical parameterization of discontinuous Galerkin method applied to wave propagation problems," Journal of Computational Physics, vol. 223, pp. 188-207, Apr. 2007.

${ }^{8}$ E. J. Kubatko, C. Dawson, and J. J. Westerink, "Time step restrictions for Runge-Kutta discontinuous Galerkin methods on triangular grids," Journal of Computational Physics, vol. 227, pp. 9697-9710, Dec. 2008.

${ }^{9} \mathrm{~T}$. Toulorge and W. Desmet, "CFL Conditions for Runge-Kutta discontinuous Galerkin methods on triangular grids," Journal of Computational Physics, vol. 230, pp. 4657-4678, June 2011.

${ }^{10}$ H. L. Atkins and D. P. Lockard, "A High-Order Method Using Unstructured Grids for the Aeroacoustic Analysis of Realistic Aircraft Configurations," AIAA, no. AIAA-99-1945, 1999.

${ }^{11}$ D. Stanescu, M. Y. Hussaini, and F. Farassat, "Aircraft engine noise scattering by fuselage and wings: a computational approach," Journal of sound and vibration, vol. 263, no. 2, pp. 319-333, 2003.

${ }^{12}$ C. P. A. Blom, Discontinuous Galerkin method on tetrahedral elements for aeroacoustics. PhD thesis, University of Twente, Enschede, 2003.

${ }^{13} \mathrm{M}$. Bernacki and S. Piperno, "A dissipation-free time-domain discontinuous Galerkin method applied to three-dimensional linearized Euler equations around a steady-state non-uniform inviscid flow," Journal of Computational Acoustics, vol. 14, no. 04, pp. 445-467, 2006.

${ }^{14}$ Y. Reymen, "3d High-Order Discontinuous Galerkin Methods for Time-Domain Simulation of Flow Noise Propagation (3d Discontinue Galerkin methoden voor simulatie in het tijdsdomein van devoortplanting van stromingsgeluid)," 2008.

${ }^{15}$ T. Toulorge, Efficient Runge-Kutta Discontinuous Galerkin Methods Applied to Aeroacoustics. PhD thesis, Ph. D. Thesis, Katholieke Universiteit Leuven, 2012.

${ }^{16}$ R. Della Ratta Rinaldi, A. Iob, and R. Arina, "An efficient discontinuous Galerkin method for aeroacoustic propagation," International Journal for Numerical Methods in Fluids, vol. 69, pp. 1473-1495, July 2012.

${ }^{17}$ M. E. Goldstein, "An exact form of Lilley's equation with a velocity quadrupole/temperature dipole source term," Journal of Fluid Mechanics, vol. 443, pp. 231-236, 2001.

${ }^{18}$ C. K. Tam and J. C. Webb, "Dispersion-Relation-Preserving Finite Difference Schemes for Computational Acoustics," Journal of Computational Physics, vol. 107, pp. 262-281, Aug. 1993.

${ }^{19}$ P. Huerre and P. A. Monkewitz, "Local and Global Instabilities in Spatially Developing Flows," Annual Review of Fluid Mechanics, vol. 22, pp. 473-537, Jan. 1990.

${ }^{20}$ J. S. Hesthaven, Nodal discontinuous Galerkin methods: algorithms, analysis, and applications. No. 54 in Texts in applied mathematics, New York: Springer, 2008.

${ }^{21}$ J. Berland, C. Bogey, and C. Bailly, "Low-dissipation and low-dispersion fourth-order Runge-Kutta algorithm," Computers $\&$ Fluids, vol. 35, pp. 1459-1463, Dec. 2006.

${ }^{22}$ G. Gabard and E. Brambley, "A full discrete dispersion analysis of time-domain simulations of acoustic liners with flow," Journal of Computational Physics, vol. 273, pp. 310-326, Sept. 2014. 